\title{
Non-equilibrium Surface Growth and Scalability of Parallel Algorithms for Large Asynchronous Systems
}

\author{
G. Korniss ${ }^{1}$, M. A. Novotny ${ }^{1}$, Z. Toroczkai ${ }^{2}$, and P. A. Rikvold ${ }^{1,3}$ \\ 1 School of Computational Science and Information Technology, \\ Florida State University, Tallahassee, Florida 32306-4120, USA \\ 2 Department of Physics, University of Maryland, \\ College Park, MD 20742-4111, USA \\ 3 Department of Physics and Center for Materials Research and Technology, \\ Florida State University, Tallahassee, Florida 32306-4350, USA
}

\begin{abstract}
The scalability of massively parallel algorithms is a fundamental question in computer science. We study the scalability and the efficiency of a conservative massively parallel algorithm for discrete-event simulations where the discrete events are Poisson arrivals. The parallel algorithm is applicable to a wide range of problems, including dynamic Monte Carlo simulations for large asynchronous systems with short-range interactions. The evolution of the simulated time horizon is analogous to a growing and fluctuating surface, and the efficiency of the algorithm corresponds to the density of local minima of this surface. In one dimension we find that the steady state of the macroscopic landscape is governed by the EdwardsWilkinson Hamiltonian, which implies that the algorithm is scalable. Preliminary results for higher-dimensional logical topologies are discussed.
\end{abstract}

\section{Introduction}

Dynamic Monte Carlo (MC) simulations are invaluable tools for investigating the evolution of complex systems. For a wide range of systems it is plausible to assume (and in rare cases it is possible to derive) that attempts to update the state of the system form a Poisson process. The basic notion is that time is continuous, and the discrete events (update attempts) occur instantaneously. The state of the system remains constant between events. It is worthwhile to note that the standard random-sequential update schemes (easily implementable on serial computers) produce this dynamics for "free:" the waiting-time distribution for the attempts to update each subsystem or component is geometrical and approaches the exponential distribution in the large-system limit. This uniquely characterizes the Poisson process.

The parallel implementation of these dynamic MC algorithms belongs to the class of parallel discrete-event simulation, which is one of the most challenging areas in parallel computing [1]. The numerous applications range from the natural sciences and engineering to computer science and queueing networks. For example, in lattice Ising models the discrete events are spin-flip 
attempts, while in queueing systems they are job arrivals. The difficulty of parallel discrete-event simulations is that update attempts are not synchronized by a global clock. In fact, the traditional dynamic MC algorithms were long believed to be inherently serial, i.e., in spin language, the corresponding algorithm was thought to be able to update only one spin at a time. However, Lubachevsky presented an approach for parallel simulation of these systems [2] without changing the underlying Poisson process. Applications include modeling of cellular communication networks [3], particle deposition [4, and metastability and hysteresis in kinetic Ising models [5].

In a distributed massively parallel scheme each processing element (PE) carries a subsystem of the full system. The parallel algorithm must concurrently advance the Poisson streams corresponding to each subsystem without violating causality. This requires the concept of local simulated time, as well as a synchronization scheme. Intuitively it is clear that systems with short-range interactions contain a "substantial" amount of parallelism. For the "conservative" approach [2, the efficiency of the algorithm is simply the fraction of PEs that are guaranteed to attempt the update without breaking causality. The rest of the PEs must idle.

\section{Time Horizon Evolution and Efficiency Modeling}

We consider a $d$-dimensional hypercubic regular lattice topology where the underlying physical system has only nearest-neighbor (nn) interactions (e.g., Glauber spin-flip dynamics) and periodic boundary conditions. The scalability analysis is made for the "worst-case" scenario in which each PE hosts a single site (e.g., one spin) of the underlying system. While this may be the only scenario for a special-purpose computer with extremely limited local memory, on architectures with relatively large memory one PE can host a block of sites. This substantially increases the efficiency, bringing it to the level of practical applicability [5].

In the basic parallel scheme [2, each PE generates its own local simulated time for the next update attempt. The set of local times $\left\{\tau_{i}(t)\right\}_{i=1}^{L^{d}}$ constitute the simulated time horizon. Here, $L$ is the linear size of the lattice $\left(L^{d}\right.$ is the number of PEs), and $t$ is the index of the simultaneously performed parallel steps. Initially, $\tau_{i}(0)=0$ for every site. At each parallel time step, only those PEs for which the local simulated time is not greater than the local simulated times of their nn can attempt the update and increment their local time by an exponentially distributed random amount, $\eta_{i}(t)$. Without loss of generality we take $\left\langle\eta_{i}(t)\right\rangle=1$. The other PEs idle. Due to the continuous nature of the random simulated times, for $t>0$ the probability of equal-time updates for any two sites is of measure zero. The comparison of the nn simulated times and idling if necessary enforces causality. Since at worst the PE with the absolute minimum simulated time makes progress, the algorithm is free from deadlock. For this basic conservative scheme, the theoretical efficiency (ignoring 

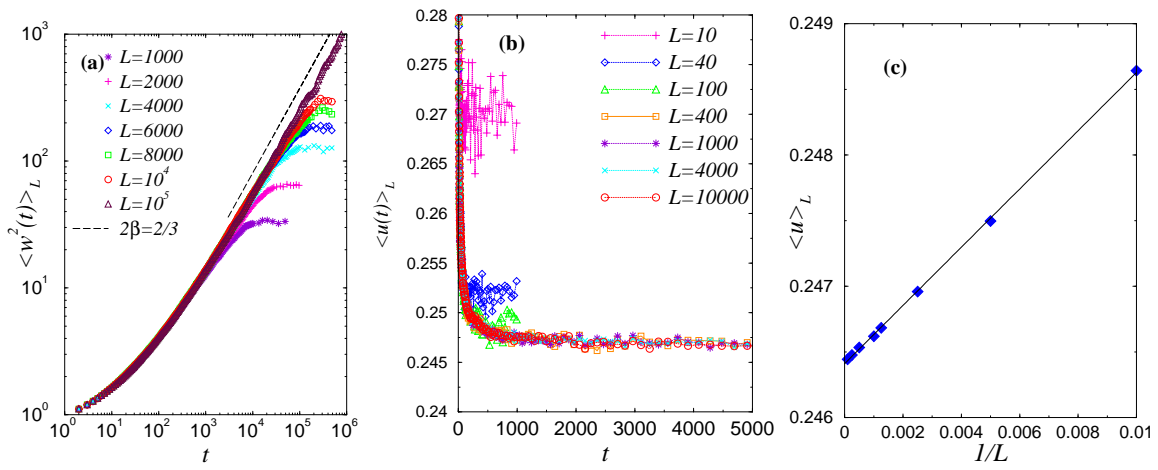

Fig. 1. Evolution of the simulated time horizon in one dimension: (a) surface width; (b) density of local minima; (c) Steady-state scaling of the parallel efficiency (density of local minima). Note the small vertical scales in (b) and (c)

communication overheads) is simply the fraction of non-idling PEs. This corresponds to the density of local minima of the simulated time horizon. Note that the evolution of the simulated time horizon is completely independent of the underlying model (except for its topology) and can be written as:

$$
\tau_{i}(t+1)=\tau_{i}(t)+\prod_{j \in D_{i}^{\mathrm{nn}}} \Theta\left(\tau_{j}(t)-\tau_{i}(t)\right) \eta_{i}(t) .
$$

Here $D_{i}^{\text {nn }}$ is the set of nearest neighbors of site $i$, and $\Theta(\cdot)$ is the Heaviside step function. The evolution of the simulated time horizon is clearly analogous to an irreversibly growing and fluctuating surface.

There are two important quantities to study. The first is the density of local minima, $\langle u(t)\rangle_{L}$, in particular its asymptotic (or steady-state) value and finite-size effects. It corresponds directly to the efficiency of the algorithm. The second is the surface width, $\left\langle w^{2}(t)\right\rangle=\left(1 / L^{d}\right)\left\langle\sum_{i=1}^{L^{d}}\left[\tau_{i}(t)-\bar{\tau}(t)\right]^{2}\right\rangle$, where $\bar{\tau}(t)=\left(1 / L^{d}\right) \sum_{i=1}^{L^{d}} \tau_{i}(t)$. It describes the macroscopic roughness of the time horizon and has important consequences for actual implementations [6] (e.g., optimal buffer size for a collecting statistics network [2]).

For $d=1$ we showed [7] by coarse-graining and direct simulation of (11) that the evolution of the simulated time horizon belongs to the KPZ universality class [8]. Our simulation confirmed that before reaching the steady state, $\left\langle w^{2}(t)\right\rangle \sim t^{2 \beta}$ with $\beta \approx 1 / 3$ [Fig. 1(a)]. At the same time the density of local minima, $\langle u(t)\rangle_{L}$, decreases monotonically with time towards a long-time asymptotic limit well separated from zero [Fig. 1(b)]. The steady state is governed by the Edwards-Wilkinson Hamiltonian [9], and the stationary width scales as $\left\langle w^{2}\right\rangle \sim L^{2 \alpha}$, where $\alpha=1 / 2$ is the roughness exponent. This guarantees that the coarse-grained landscape is a simple random-walk surface; the local slopes are short-range correlated. Thus, the density of local minima is non-zero. The non-zero density of local minima is a universal characteristic of 

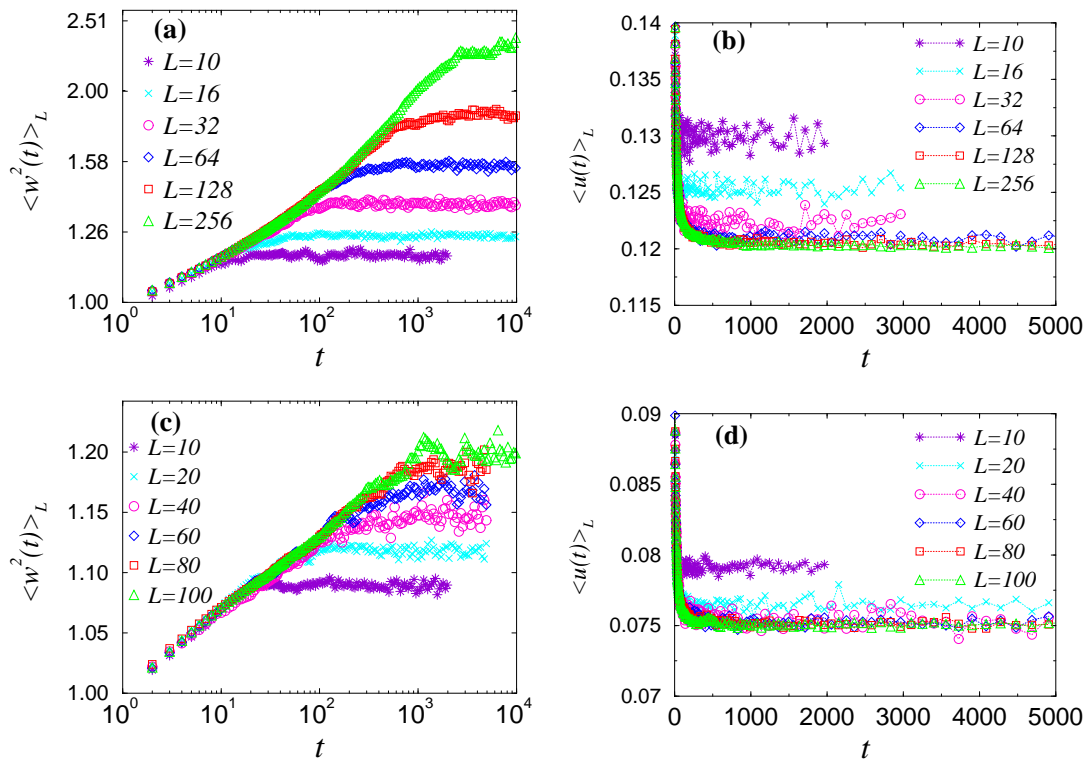

Fig. 2. Evolution of the simulated time horizon in $d=2$ and $d=3$ : (a) surface width in $d=2$; (b) density of local minima in $d=2$; (c) surface width in $d=3$; (d) density of local minima in $d=3$.

this class [7, 10]. Further, its steady-state finite-size effects can be written as $\langle u\rangle_{L}=\langle u\rangle_{\infty}+$ const $/ L$. The $\mathcal{O}(1 / L)$ correction in $d=1$ appears to be rather robust for periodic boundary conditions [10]. The extrapolated value for the efficiency is $\langle u\rangle_{\infty}=0.2464(1)$ [Fig. 1(c)].

In higher dimensions we observe the same qualitative behavior as for $d=1$. The surface roughens and saturates for any finite system, as seen in Fig. 2(a,c). Simultaneously, the density of local minima decreases monotonically towards its asymptotic $(t \rightarrow \infty)$ finite-size value [Fig. 2(b,d)]. Again, the steady-state density of local minima appears to be well separated from zero. For $d=2\langle u\rangle_{\infty} \approx 0.12$, and for $d=3\langle u\rangle_{\infty} \approx 0.075$. The $\langle u\rangle_{\infty} \sim \mathcal{O}(1 / K)$ behavior appears to be rather general [6], where $K=2 d$ is the number of nearest neighbors on a regular lattice.

Similar to the $d=1$ case, corrections to scaling are very strong, both for the surface width and the density of local minima. While for $d=1$ we were able to simulate large systems $\left(L \gg 10^{3}\right)$ to obtain the KPZ scaling exponents and the steady-state finite-size behavior of $\langle u\rangle_{L}$, in higher dimensions the relatively small system sizes prevented us from extracting the scaling behavior of the width and the finite-size effects of the density of local minima.

We conjecture that the simulated time horizon exhibits KPZ-like evolution in higher dimensions as well. While the scalability of the parallel scheme for random topology has been investigated earlier [6], the underlying mechanism 
for regular lattices (macroscopic roughening) was only recently pointed out [7]. The major implication is that while the algorithm is scalable, and the width of the simulated time horizon saturates for any finite number of PEs, it diverges in the limit of an infinite number of PEs. Thus, in an actual implementation the programmer has to take some actions to handle statistics collection.

\section{Summary and Outlook}

The analogy between the evolution of the simulated time horizon and nonequilibrium surface growth illustrates that when devising parallel algorithms, the programmer has to be very much concerned with the morphological properties of the associated surface. To fully describe the evolution of the simulated time horizon and the efficiency of the algorithm, one must focus on both the long wave-length behavior (the macroscopic width) and short-distance properties (the density of local minima). While most previous surface studies focus on the long wave-length properties, physics at short distances is just as challenging, has real applications, and may reveal universal features [7].10].

\section{Acknowledgments}

We thank B. D. Lubachevsky, A. Weiss, and S. Das Sarma for useful discus-

sions. We acknowledge the support of DOE through SCRI-FSU, NSF-MRSEC at UMD, and NSF through Grant No. DMR-9871455.

\section{References}

1. R. M. Fujimoto, Commun. ACM 33, 30 (1990)

2. B. D. Lubachevsky, Complex Systems 1, 1099 (1987); J. Comput. Phys. 75, 103 (1988)

3. A. G. Greenberg, B. D. Lubachevsky, D. M. Nicol, and P. E. Wright, Proceedings, 8th Workshop on Parallel and Distributed Simulation (PADS '94), Edinburgh, UK, (1994) p. 187

4. B. D. Lubachevsky, V. Privman, and S. C. Roy, J. Comput. Phys. 126, 152 (1996)

5. G. Korniss, M. A. Novotny, and P. A. Rikvold, J. Comput. Phys., 153, 488 (1999)

6. A. G. Greenberg, S. Shenker, and A. L. Stolyar, Performance Evaluation Review 24, 91 (1996)

7. G. Korniss, Z. Toroczkai, M. A. Novotny, and P. A. Rikvold, Phys. Rev. Lett. 84, $1351(2000)$

8. M. Kardar, G. Parisi, and Y.-C. Zhang, Phys. Rev. Lett. 56, 889 (1986)

9. S. F. Edwards and D. R. Wilkinson, Proc. R. Soc. London, Ser A 381, 17 (1982)

10. Z. Toroczkai, G. Korniss, S. Das Sarma, and R. K. P. Zia, submitted to Phys. Rev. E; e-print cond-mat/0002143 (2000) 